\title{
History Matching and Production Forecast Uncertainty by Means of the Ensemble Kalman Filter: a Real Field Application
}

\author{
Bianco, A., Cominelli, A., Dovera, L. (Eni E\&P Division), Naevdal, G., Valles, B. (Iris)
}

Copyright 2007, Society of Petroleum Engineers

This paper was prepared for presentation at the SPE Europec/EAGE Annual Conference and Exhibition held in London, United Kingdom, 11-14 June 2007.

This paper was selected for presentation by an SPE Program Committee following review of information contained in an abstract submitted by the author(s). Contents of the paper, as presented, have not been reviewed by the Society of Petroleum Engineers and are subject to correction by the author(s). The material, as presented, does not necessarily reflect any position of the Society of Petroleum Engineers, its officers, or members. Papers presented a SPE meetings are subject to publication review by Editorial Committees of the Society of Petroleum Engineers. Electronic reproduction, distribution, or storage of any part of this pape for commercial purposes without the written consent of the Society of Petroleum Engineers is prohibited. Permission to reproduce in print is restricted to an abstract of not more than 00 words; illustrations may not be copied. The abstract must contain conspicuous 300 words; illustrations may not be copied. The abstract must contain conspicuous

acknowledgment of where and by whom the paper was presented. Write Librarian, SPE, P.O.
Box 833836, Richardson, Texas 75083-3836 U.S.A., fax 01-972-952-9435.

\section{Abstract}

During history match reservoir models are calibrated against production data to improve forecasts reliability. Often, the calibration ends up with a handful of matched models, sometime achieved without preserving the prior geological interpretation. This makes the outcome of many history matching projects unsuitable for a probabilistic approach to production forecast, then motivating the quest of methodologies casting history match in a stochastic framework.

The Ensemble Kalman Filter (EnKF) has gained popularity as Monte-Carlo based methodology for history matching and real time updates of reservoir models. With EnKF an ensemble of models is updated whenever production data are available. The initial ensemble is generated according to the prior model, while the sequential updates lead to a sampling of the posterior probability function.

This work is one of the first to successfully use EnKF to history match a real field reservoir model. It is, to our knowledge, the first paper showing how the EnKF can be used to evaluate the uncertainty in the production forecast for a given development plan for a real field model. The field at hand was an on-shore saturated oil reservoir. Porosity distribution was one of the main uncertainties in the model, while permeability was considered a porosity function.

According to the geological knowledge, the prior uncertainty was modeled using Sequential Gaussian Simulation and ensembles of porosity realizations were generated. Initial sensitivities indicated that conditioning porosity to available well data gives superior results in the history matching phase. Next, to achieve a compromise between accuracy and computational efficiency, the impact of the size of the ensemble on history matching, porosity distribution and uncertainty assessment was investigated. In the different ensembles the reduction of porosity uncertainty due to production data was noticed. Moreover, EnKF narrowed the production forecast confidence intervals with respect to estimate based on prior distribution.

\section{Introduction}

Reservoir management of modern oil and gas fields requires periodic updates of the simulation models to integrate in the geological parameterization production data collected over time. In these processes the challenges nowadays are many. First, a coherent view of the geomodel requires updating the simulation decks in ways consistent with geological assumptions. Second, the management is requiring more and more often a probabilistic assessment of the different development scenarios. This means that cumulative distribution functions, reflecting the underlying uncertainty in the knowledge of the reservoir, for key production indicators, e.g. cumulative oil production at Stock Tank condition (STC), along the entire time-life of the field, are expected outcomes of a reservoir modeling project. Moreover, production data are nowadays collected with increasing frequencies, especially for wells equipped with permanent down-hole sensors. Decision making, based on most current information, requires frequent and rapid updates of the reservoir models.

The Ensemble Kalman Filter (EnKF) is a Monte-Carlo based method developed by Evensen ${ }^{1}$ to calibrate oceanographic models by sequential data assimilation. Since the pioneering application on near-well modeling problems by Naevdal et al. ${ }^{2}$, EnKF has become in the reservoir simulation community a popular approach for history matching and uncertainty assessment ${ }^{3-7}$. This popularity is motivated by key inherent features of the method.

EnKF is a sequential data assimilation methodology, and then production data can be integrated in the simulation model as they are available. This makes EnKF well suited for realtime application, where data continuously collected have to be used to improve the reliability of predictive models.

EnKF maintains a Gaussian ensemble of models aligned with the most current production data by linear updates of the model parameters. In that way the statistical properties of the Gaussian ensemble, that is to say mean, variance and twopoint correlations are preserved.

Because EnKF does not need either history matching gradients or sensitivity coefficients, any reservoir simulator with restarting capabilities can be used in an EnKF workflow, without modifying simulator source code. This represents an obvious advantage with respect to methods like the 
Randomized Maximum Likelihood (RML) method ${ }^{8,9}$, which requires a simulator with adjoint gradient capabilities ${ }^{11}$.

These reasons motivate the interest on EnKF in the Upstream Industry. Nonetheless, only a few real applications were published before this work $^{12,13}$. Skjervheim et. al. ${ }^{12}$ compared results on using EnKF to assimilate 4D seismic data and production data, and obtained results that slightly improved the base case used for comparison. Haugen et al., see Ref. 13, report that the EnKF was used to successfully history match the simulation model of a Northern sea field, with substantial improvement compared to the reference case.

In this paper we applied EnKF to history match the Zagor simulation model, quantifying also the reduction of uncertainty due to the assimilation of the production data. Different ensembles were used to investigate the connection between the effectiveness of EnKF and the size of the statistical samples. Next, we used one of the ensembles updated with EnKF to assess the uncertainty in the production forecasts. To our knowledge, this is the first paper where EnKF was used on a real reservoir from history match to uncertainty analysis of production forecasts.

The paper proceeds as follows. The next section is dedicated to the discussion of the EnKF methodology, including its mathematical background and some remarks on the current limitations. Then the Zagor reservoir model is described. That includes the geological parameterization used in this work and the presentation of the different ensembles utilized in the application. The results of the application are presented in two subsequent sections. The first is dedicated to history matching and the second dedicated to the assessment of the uncertainty in the production forecasts. Finally, conclusions based on our results are drawn and some perspectives for future works are given.

\section{The Ensemble Kalman Filter}

The EnKF is a statistical methodology suitable to solve inverse problem, especially in cases where observed data are available sequentially in time.

Assuming that the evolution of a physical system can be approximated by a numerical model, typically by the discretisation of a partial differential equation, a state vector can be used to represent the model parameters and observations. Using multiple realizations of the state vector one is able to explicitly express the model uncertainty. The EnKF can describe the evolution of the system by updating the ensemble of state vectors whenever an observation is available.

In reservoir simulation, EnKF can be applied to integrate production data by updating sequentially an ensemble of reservoir models during the simulation. Each reservoir model in the ensemble is kept up-to-date as production data are assimilated sequentially.

In this context every reservoir state vector comprises three types of parameters: static parameters, dynamic parameters and production data.

The static parameters are the parameters that in traditional history matching do not vary with time during a simulation, such as permeability $(K)$ and porosity $(\phi)$.

The dynamic parameters include the fundamental variables of the flow simulation. These are, for black oil models, the cell pressure $(p)$, water saturation $\left(S_{w}\right)$, gas saturation $\left(S_{g}\right)$ and solution gas-oil ratio $(R S)$. In addition to the variables for each cells one add observations of the production data in each well. Production data usually include simulated data corresponding to observations such as well production rates, bottom-hole pressure values, water cut (WCT) and gas oil ratio (GOR) values. Thus, using the notation by $\mathrm{X}$. H. Wen and W. H. Chen $^{6}$, the ensemble of state variables is modelled by multiple realizations:

$$
y_{k, j}=\left[\begin{array}{c}
m_{s} \\
m_{d} \\
d
\end{array}\right]_{k, j}
$$

where $y_{k, j}$ is the $j^{\text {th }}$ ensemble member of the state vector at time $t_{k}, m_{s}$ and $m_{d}$ are the static and dynamic variables respectively, and $d$ is the production data vector.

The EnKF evolves the ensemble of state variables in time assimilating the most current observations of production data by updating both static and dynamic part of all the realizations to honour the observations. This means that an ensemble of updated reservoir models is always available and statistics about the model uncertainty can be explicitly expressed.

In particular the filter consists of two processes for each time step: the forecast process, based on the current state variable, and the analysis process, including data assimilation and updating of the state variables. In the forecast step each model is simulated until the next observation date.

The state variables are advanced in time as:

$$
y_{k, j}^{f}=F\left(y_{k-1, j}^{u}\right) \quad j=1,2, \ldots N_{e}
$$

where $F$ is the forward model, $y_{k, j}^{f}$ the $j$-th state vector after running the $k$-th forecasting step and $N_{e}$ is the number of ensemble members. The superscript $f$ denotes forecast, meaning that the values are output from the simulator before Kalman Filter updating. Let $H_{k}$ be a matrix operator that relates the state vector to the production data. The production data are part of the state vector as shown in Eq. (1), so $H_{k}$ is in the form:

$$
H_{k}=[0 \mid I]
$$

where 0 is a $N_{d, k} \times\left(N_{y, k}-N_{d, k}\right)$ matrix with all 0 's as its entries; $I$ is an $N_{d, k} \times N_{d, k}$ identity matrix. Let $C_{d, k}$ be the covariance matrix of the measurement noise with dimension $N_{d, k} \times N_{d, k}$, which is diagonal if the production data errors are independent. The matrix $C_{y, k}^{f}$ defines the covariance for the state variables at time $t_{k}$ that can be estimated from the ensemble of forecasted results $\left(y_{k, j}^{f}\right)$ using the standard statistical method:

$$
C_{y, k}^{f}=\frac{1}{N_{e}-1} \sum_{j=1}^{N_{e}}\left(Y_{k, j}^{f}-\bar{Y}_{k}^{f}\right)\left(Y_{k, j}^{f}-\bar{Y}_{k}^{f}\right)^{T}
$$


where $Y_{k, j}^{f}$ is the $\mathrm{j}^{\text {th }}$ ensemble member of forecasted state vector at time $t_{k}$ with dimension of $N_{y, k} \cdot \bar{Y}_{k}^{f}$ is the mean of the state variable which is a vector of dimension $N_{y, k}$.

Then the Kalman gain $K_{k}$ can be computed as:

$$
K_{k}=C_{y, k}^{f} H_{k}^{T}\left(H_{k} C_{y, k}^{f} H_{k}^{T}+C_{d, k}\right)^{-1}
$$

Under the assumption that $y_{k, j}$ is Gaussian distributed, a variance minimizing scheme is used to update the state vector according to the observed production data, $d_{k, j}$. In particular, by using the Kalman gain $K_{k}$ as weighting matrix, the state variables become

$$
y_{k, j}^{u}=y_{k, j}^{f}+K_{k}\left(d_{k, j}-H_{k} y_{k, j}^{f}\right)
$$

The last equation shows that the updating of the forecast vector, denoted by the superscript $\mathrm{u}$, depends on the difference between simulated and observed production data; the larger the difference, the larger update will be applied to the initial state vector.

The covariance matrix, after being updated, can be assessed as:

$$
C_{y, k}^{u}=\left(I-K_{k} H_{k}\right) C_{y, k}^{f}
$$

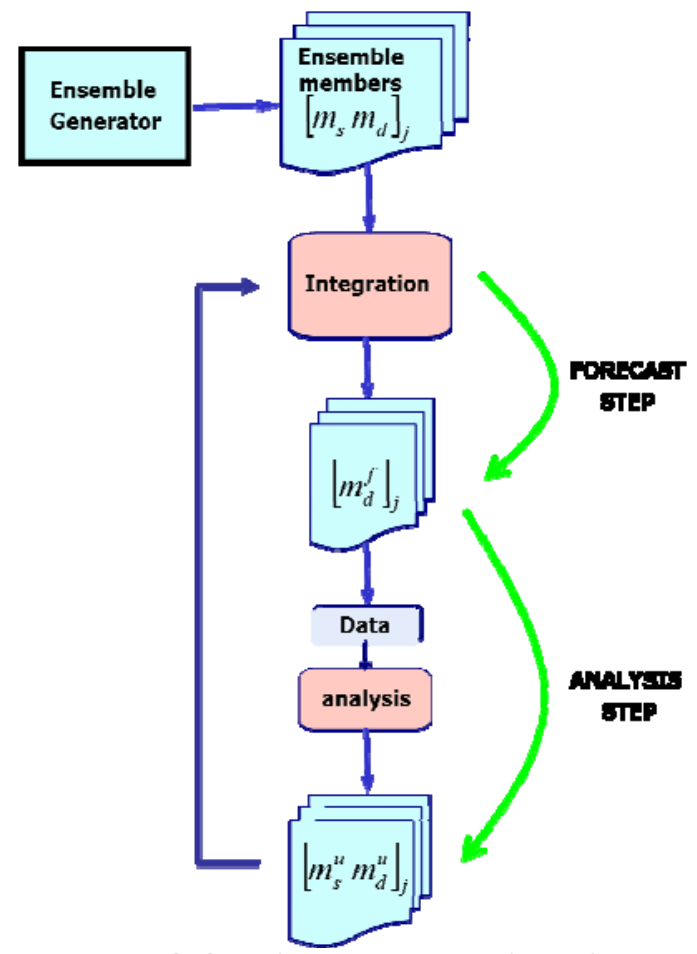

Figure 1: Description of the overall workflow of the EnKF where $m_{d}$ is the model state vector (pressure, saturation, etc.) and $m_{s}$ is the model parameter vector (porosity and permeability).

Using the updated covariance matrix, the state vector of each realization in the ensemble should reflect the most current observations $\left(d_{k}\right)$, then the procedure can be applied again till the next time step when new observations are available for assimilation.
Based on our assumption that $y$ is Gaussian distributed, the validity of Eq. (5) and Eq. (6) relies only on the assumptions that the model error and observation error are independent and also both model and observation errors are uncorrelated in time; a linear relationship between data and model is not required. This means that even if the forward model is highly non-linear, the EnKF preserves the spatial correlations of the updated models, based on the initial variogram, and the original geological interpretation is retained.

The EnKF workflow is outlined in Figure 1. It is not intended in this paper to give a thorough discussion of the foundation of the methods. The interested readers may refer to the recent book by G. Evensen ${ }^{17}$.

\section{The Zagor Reservoir}

The EnKF methodology was applied to history match the Zagor Field simulation model, using for this purpose different statistical ensembles. One of these ensembles was then used to evaluate the uncertainty in production forecast associated to given development projects.

The Zagor field is an on-shore oil field located in West Africa. The field consists of a $32 \mathrm{ft}$ thick sand body limited on top by a shale cap. A saddle in the structure separates the East side of the reservoir, well known because of the large number of drilled wells, from the less known West side. The reservoir was originally in saturated conditions, with a primary gas cap on top of a thin oil rim in the East portion, while in the West portion a gas-water contact was established by geophysical consideration, see Figure 2. The field is bounded by faults on North, East and South, while an edge aquifer acts on the West flank. Eight appraisal wells plus two production wells were drilled in the field. Gas-oil and water-oil contacts were originally at $8947.3 \mathrm{ft}$ and $9116.3 \mathrm{ft}$ TVD, respectively.

According to log data and inter well correlations, seven geological layers were identified. The seven layers were made of good quality sands with porosity log values ranging from $10 \%$ to $24 \%$. No flow barriers for vertical flow were recognized.

The field was simulated using a black-oil model. The fluids can be characterized by means of an oil gravity of $37^{\circ} \mathrm{API}$, an oil formation volume factor of $1.5 \mathrm{RB} / \mathrm{STB}$, an oil viscosity of $0.28 \mathrm{cp}$ and an undersaturated compressibility of $1.35 \times 10^{-5} \mathrm{psia}^{-1}$. The initial bubble point pressure was 3885 psia, corresponding to a gas-oil ratio (GOR) of $1 \mathrm{Mscf} / \mathrm{STB}$ in the oil rim. The simulation grid consisted of $156 \times 77 \times 10$ corner point geometry cells, with a lateral spacing of 385 feet $x 385$ feet. As regards the thickness of the cells a value of 3 feet, with some small variation between layers, represents the average cell thickness. Notably, the simulation layering results from a refinement of some layers: geological layer 2, 5 and 7 were split into sub-layers. A total of 25669 cells were active in the simulation.

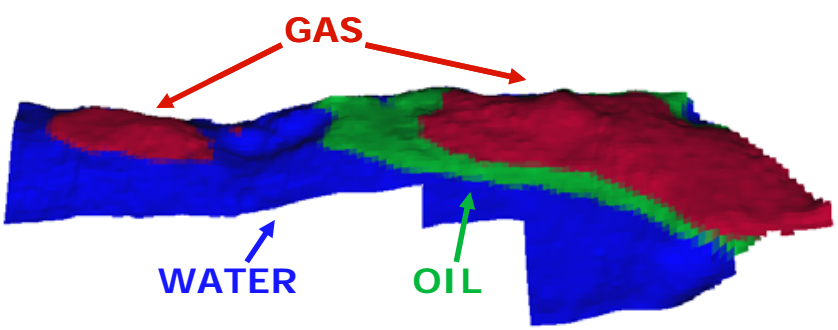


Figure 2: Zagor field original phase distribution.

The relative permeability functions of the oil-water and gas-water systems are shown in Figure 3.
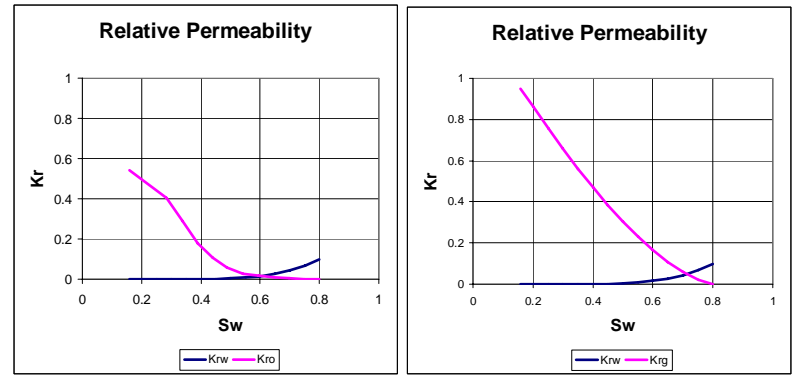

Figure 3: Oil-water, left, and gas-oil relative permeability, right.

\section{Definition of Baseline Geological Model}

The key issue in the geological parameterization was the definition of porosity values in the simulation grid. A baseline geological model was defined by using $\log$ porosity values available in the ten wells drilled in the field. These values were utilized to condition a sequence of seven maps, one per geological layer. Kriging was used to propagate the wells porosity values throughout the whole reservoir, with standard deviation derived on a layer basis according to the available $\log$ data. A Kriged porosity map for a representative layer is shown in Figure 4.

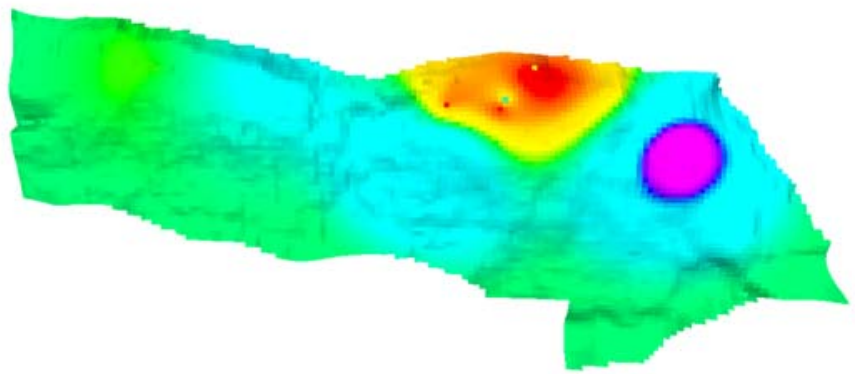

Figure 4: Kriged porosity map in a representative layer of the Zagor field. Red means high porosity values (up to $25 \%$ ), violet means very low (down to $2.5 \%$ ) values.

Both the geological and the simulation grid had the same lateral spacing. Then, porosity values generated in the geological model could be copied from the geological grid to the simulation grid.

According to the geological analysis of core and log data, a deterministic functional dependency between permeability and porosity was assumed ${ }^{10}$. This, together with a vertical to horizontal permeability ratio of 1 , led to the definition of the static parameters of the simulation grid. Initial saturation values were set according to relative permeability end-points.

\section{Generation of Gaussian Ensembles}

EnKF updates sequentially ensemble of Gaussian models. In this work, ensembles of simulation models were obtained by generating a sequence of porosity fields at the simulation model scale. For this purpose the Sequential Gaussian simulation (SGSim) algorithm ${ }^{14}$ was used to generate porosity fields, suitable for numerical simulation. Each porosity field was assumed to be correlated using an isotropic bi- dimensional variogram with a range of $4000 \mathrm{~m}$. Different from the study performed in Ref.13, the porosity distribution was constrained at the observed porosities in the appraisal wells.

The stochastic modeling was used to generate 135 porosity fields, to be used in the following application of EnKF methodology. Three ensembles were used: a small size ensemble, consisting of 50 models, a medium size ensemble, consisting of 100 models, and a - relatively - large size ensemble of 135 models. These three ensembles are referred hereafter as A, B and C, with 50, 100 and 135 members respectively.

The availability of differently sized ensembles allowed for evaluating the impact of the size of the ensemble of models. In previous studies on updating reservoir simulation models using ensemble Kalman filter, the number of ensemble members used varies from 40 (Ref.7) to 400 (Ref. 6). Since the first paper in this area ${ }^{2}$, it has been quite common to work with 100 ensemble members. The selection of this ensemble size was based on reported experience from large-scale problems within atmospheric sciences ${ }^{15}$. For a 2-D synthetic 2-phase five spot example, where the permeability field was estimated ${ }^{6}$, it was concluded that a relatively large ensemble is required to accurately estimate the uncertainty of the estimated fields, but that a smaller ensemble suffice to create reservoir models that match the up-to-date production data only. Simultaneous estimation of permeability and porosity fields where considered on a small size reservoir engineering model (PUNQ-S3), and it was concluded that solutions with a reasonable history match were obtained with only 40 ensemble members ${ }^{7}$. Although the dimension of the PUNQ-S3 model is small compared to realistic field models (at least one order of magnitude), it has been reported that an ensemble with 100 members is sufficient, to obtain solutions with a reasonable history match, for field cases having the same order of grid cells as the model used in the present study (see Ref. 13). A more thorough study of the effect of the ensemble size $^{16}$ concluded that a modest size is required to get models that provide history matched solution, but that a much larger ensemble size is required in order to quantify the uncertainty in the estimated static fields. This study was performed on a simplified model (the heat-equation) and should have bearing for reservoir simulation models also. Another study using the PUNQ-S3 model ${ }^{18}$ showed that 100 ensemble members was not enough to get constant uncertainty in the production forecasts. Nonetheless, further studies are required to conclude on the exact ensemble sizes required. We will see that the results obtained here are in accordance with the previous findings.

\section{History Matching the Zagor Field by Using ENKF}

The EnKF workflow described previously, equations (1) to (7), was coded in a flexible Matlab toolbox, then implemented to history match the Zagor reservoir model. In our case, the state vectors (cf. Equation 1) were composed of: porosity values as static variables, cell pressure, water saturation, gas saturation and solution gas-oil ratio as dynamic variables. The production data vector comprised the wells production rates, bottom-hole pressures, water cuts (WCT) and gas-oil ratios (GOR). In the history matching phase the three differently sized ensembles A, B, and $\mathrm{C}$ were used. This allowed for analysing the impact of the ensemble size on the history match. In the next section, available production data are first 
reviewed and the errors used to define misfits between simulation and observations are discussed.

\section{Production Data for EnKF Based History Matching}

The Zagor field was brought on stream for production by means of two vertical wells, namely well A1 and A2, completed in the oil rim. The history matching period covers approximately three years. In this period only Stock Tank condition (STC) gas, oil and water rates were collected. The history phase was simulated by using daily STC oil rates, averaged on monthly basis, as well targets. Monthly averaged GOR and WCT data were used as calibration data in the matching. GOR and WCT history data for both wells are shown in Figure 5 and Figure 6, respectively. It is worth noticing that well A2 produced under anhydrous conditions for most of the history period.

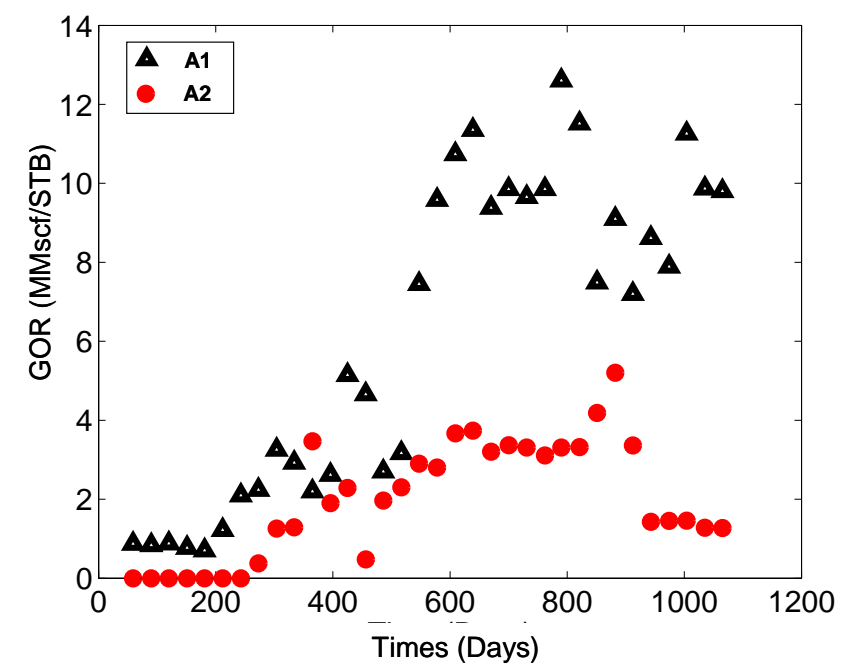

Figure 5: GOR measured data for well A1 (triangle) and A2 (circle).

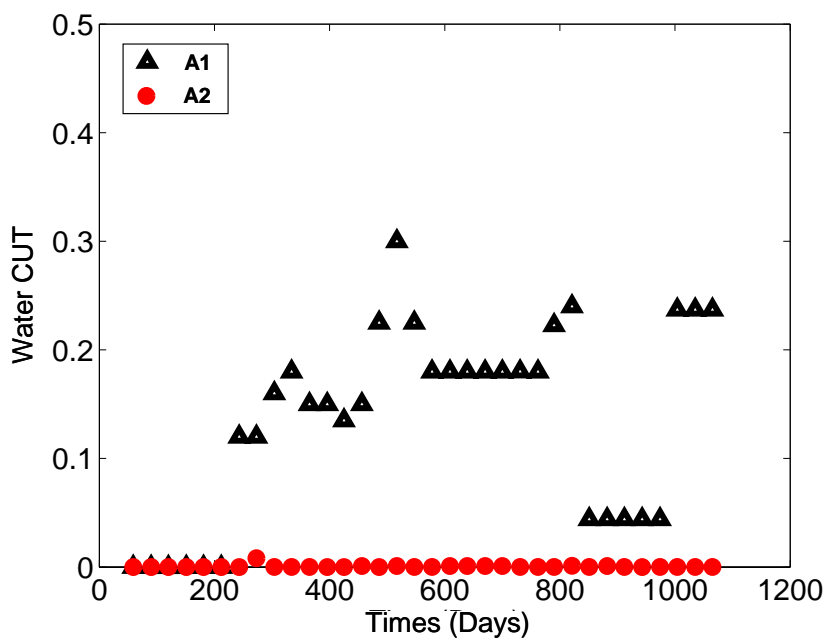

Figure 6: WCT measured data for well A1 (triangle) and A2 (circle).

The reliability of the observed data was an issue. According to the asset engineers, low GOR values were less reliable than high GOR values. Moreover, increasing GOR trends are more reliable than oscillating GOR values. This makes GOR values available for well A2 less reliable than the corresponding values for well A1. In addition, the decrease of well A2 GOR from a value of $4 \mathrm{MScf} / \mathrm{STB}$ to a plateau value of $1.5 \mathrm{MScf} / \mathrm{STB}$ in the last months of the history period was deemed pretty unreliable. As regards WCT, see Figure 6, very low - less than 0.025 - values in the time-period between day 851 and day 954 for well A1, were considered unreliable.

The engineering interpretation of the production data was used, see Table 1 , to define the errors or prior standard deviations required to weight the observations.

\begin{tabular}{|l|l|l|}
\hline & \multicolumn{1}{|c|}{ A1 } & \multicolumn{1}{c|}{ A2 } \\
\hline \multirow{3}{*}{ GOR } & $0.4 \mathrm{MScf} / \mathrm{STB}$ for GOR & $0.6 \mathrm{MScf} / \mathrm{STB}$ for \\
& values $\geq 6 \mathrm{MScf} / \mathrm{STB}$, & GOR values gathered \\
& $0.2 \mathrm{MScf} / \mathrm{STB}$ for lower & till day $882,600 \%$ for \\
& values & later time values. \\
\hline \multirow{3}{*}{ WCT } & $\begin{array}{l}0.05 \text { for values outside } \\
\text { low-reliability time } \\
\text { window, } \\
\\
\text { 600\% inside }\end{array}$ & \\
\hline
\end{tabular}

Table 1: errors used to weight the observations available for the Zagor field.

\section{Comparative Analysis of EnKF History Matching Results}

EnKF was applied to history match the three available ensembles $\mathrm{A}, \mathrm{B}$, and $\mathrm{C}$. The integration proceeded for 33 assimilation time periods, with the usual sequence of forecast and analysis steps.

The result of EnKF integration can be evaluated in many ways. In real-time problems it is interesting to investigate the effectiveness of EnKF primarily by looking at the evolution of the forecasted quantities $y_{k, j}^{f}$ along integration. This approach is effective because in a real-time application the main interest is the improvement in short-term predictive value of the ensemble. Similar considerations hold for ocean modelling.

In this application, the effectiveness of EnKF calibration was first evaluated by using the porosity fields given by the last assimilation step to simulate the history period from day 1 to day 1065 . The results of these simulations could then be compared with the results of the simulation based on the original porosity fields.

Figure 7 to Figure 9 show Well A1 GOR computed by means of EnKF porosity (blue) and by original porosity (red) for the different ensembles A, B, and C, respectively. Observed data are included in the pictures. 


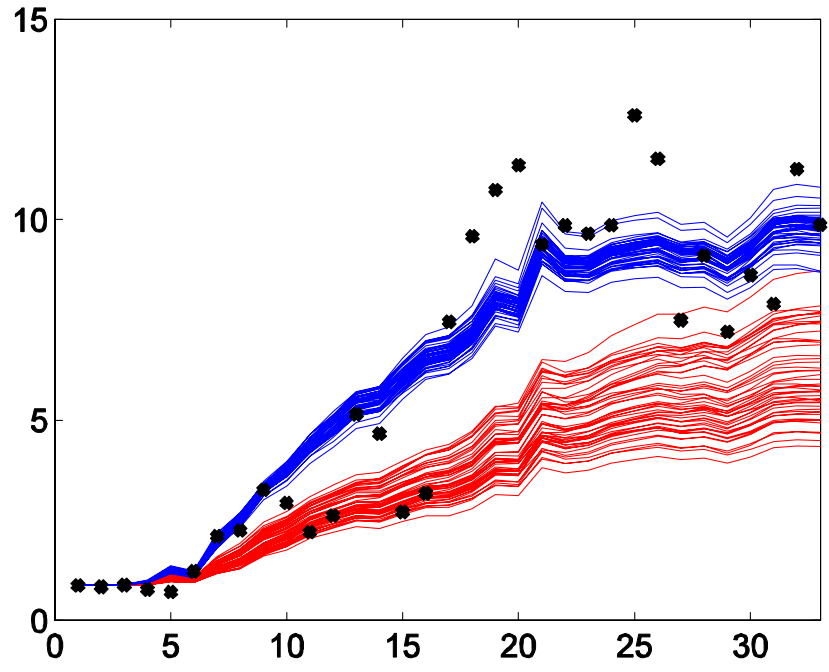

Figure 7: Well A1 GOR values: observed (bullets), computed by using the original ensemble $A$ (red) and computed by using the last EnKF updated ensemble $A$ (blue). The time unit in abscissa is one month.

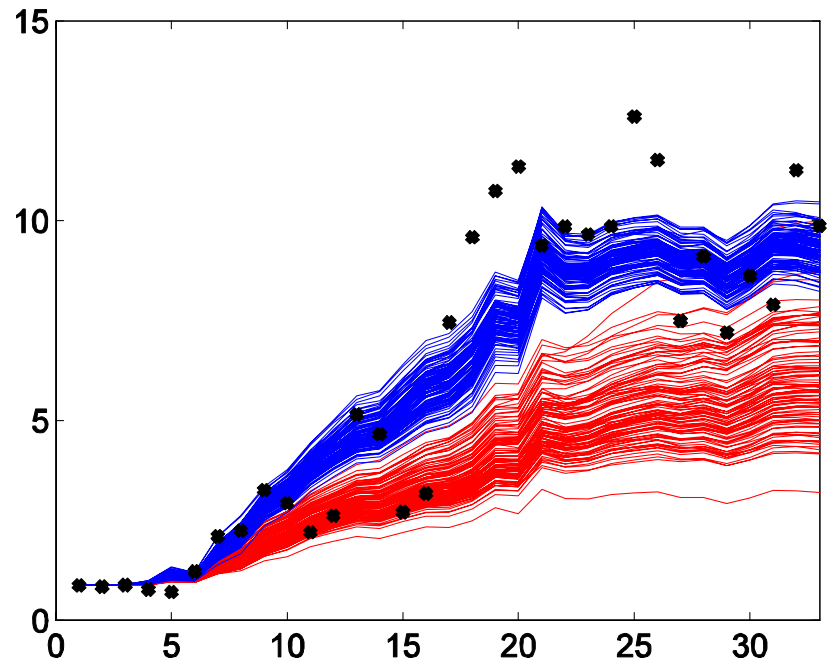

Figure 8: Well A1 GOR values: observed (bullets), computed by using the original ensemble $B$ (red) and computed by using the last EnKF updated ensemble B (blue). The time unit in abscissa is one month.

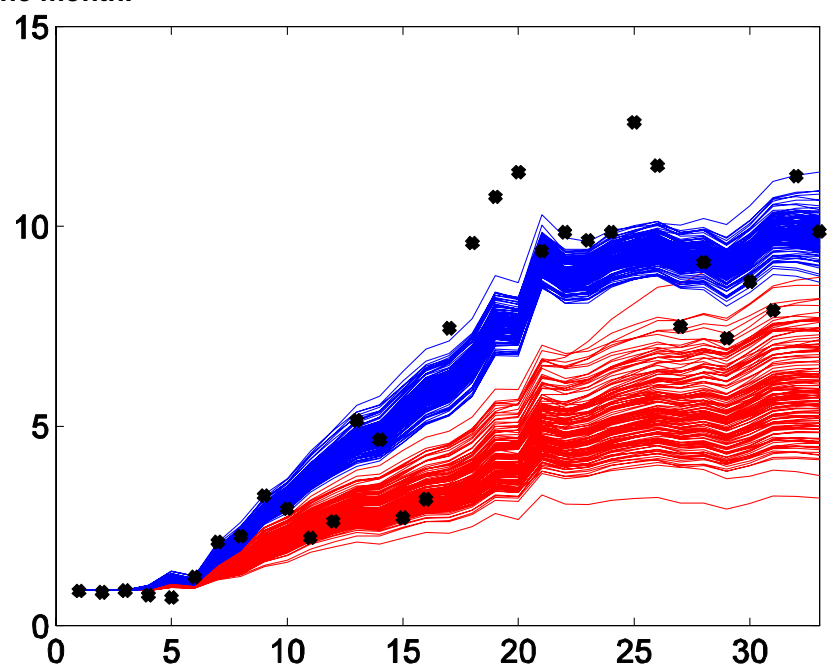

Figure 9: Well A1 GOR values: observed (bullets), computed by using the original ensemble $C$ (red) and computed by using the last EnKF updated ensemble $C$ (blue). The time unit in abscissa is one month.
At a glance, the results shown in the three previous pictures gave qualitative indications about the effectiveness of EnKF as a history matching tool. The assimilation process was able to move the envelope of the simulated data towards the observed data trend, narrowing at the same time the spread of the simulated values. Similar conclusions could be drawn by looking at the corresponding plots for well A2 GOR and WCT and well A1 WCT.

The evaluation on history match performances could be made more quantitative by computing the ensemble averaged objective function $J$ defined as

$$
J_{A}=\frac{1}{2 N_{e}} \sum_{j=1}^{N_{e}} \sum_{i=1}^{N_{o b s}}\left(\frac{o_{i}-c_{i j}}{\sigma_{i}}\right)^{2}
$$

Where $o_{i}$ and $\sigma_{i}$ are respectively the observed values GOR and WCT for the two wells - and the standard deviation for observation $\mathrm{i}$, while $c_{i j}$ is the value, given by the simulation of the $j$-th ensemble member, corresponding to the observation $o_{i}$. The standard deviations were defined according to Table 1 .

In Figure 7-9, the ensembles average objective functions values were reported for the three ensembles before and after the EnKF integration.

The table clearly indicates that the objective function was systematically decreased by EnKF, with some considerable improvement for larger ensembles.

\begin{tabular}{|l|l|l|}
\hline \multirow{2}{*}{ Ensemble } & \multicolumn{2}{|c|}{ Avg. Objective Function $J$} \\
\cline { 2 - 3 } & Before EnKF & After EnKF \\
\hline A & 1136.8 & 307.9 \\
\hline B & 1193.1 & 270.8 \\
\hline C & 1196.9 & 273.2 \\
\hline
\end{tabular}

Table 2: average objective function values for the three ensemble before and after EnKF integration.

\section{Statistical Analysis of Integrated Ensembles}

The EnKF is a Monte-Carlo based method, aimed at sampling the posterior probability and updating the covariance and the mean of the original ensemble. In this section the results of the EnKF integration are reviewed to analyse the modifications operated by EnKF on the statistical structure of the ensembles. Two statistical estimators were considered: ensemble mean and standard deviation.

\section{Ensemble Mean Updates}

The ensemble mean was defined by computing an average porosity field $\left\langle m_{s}\right\rangle_{a}^{o / u}$, where

$$
\left\langle m_{s}\right\rangle_{a}^{o / u}=\frac{1}{N_{e}} \sum_{j=1}^{N_{e}} m_{s, j}^{o / u},
$$

In equation (9) the mean field can be computed using the original ensemble (superscript $o$ ) or the ensemble after EnKF updates (superscript $u$ ), where $a$ labels the ensemble ( $a=A, B$, or $C$ ).

The average porosity fields $\left\langle m_{s}\right\rangle^{o}$ are simply approximations of the Kriged baseline porosity field, as it can 
be noticed by comparing average porosity field for the original ensemble A (Figure 10) with the corresponding Kriged porosity map (Figure 4 ).

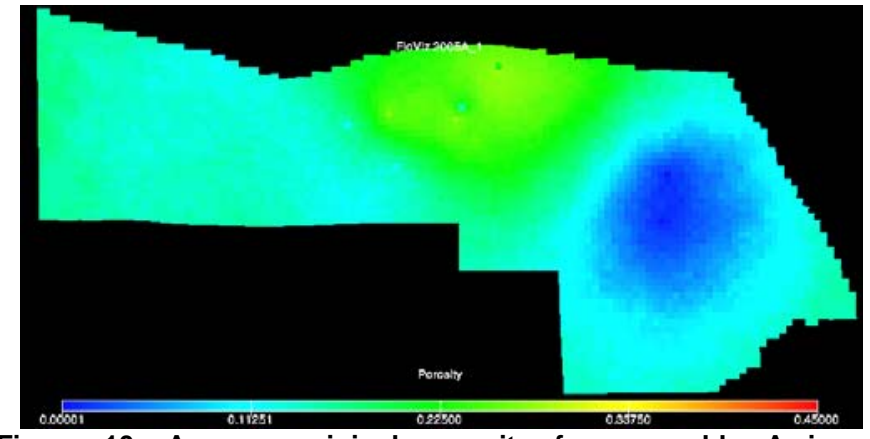

Figure 10: Average original porosity for ensemble $A$ in a representative layer.

On the contrary, the porosity fields $\left\langle m_{s}\right\rangle_{a}^{u}$, with $a=A, B$, or $C$, are unbiased estimator of the mean of the posterior distribution function. In Figure 11, Figure 12 and Figure 13 porosity maps for a representative layer are shown for ensembles $A, B$, and $C$, respectively.

By analyzing these maps, in conjunction with the Kriged porosity map shown in Figure 10, it can be noticed that EnKF integration increased the average porosity in the West and South-East flanks. Some very high values - up to $42 \%$ - may be observed in the case of ensembles A and B. In the case of ensemble $\mathrm{C}$, the porosity values span approximately the same range of the values in the original mean field, see Figure 11. The lack of porosity over-shooting might indicate that the larger ensemble led to more consistent results. Nonetheless, the over-shooting occurs mainly in the far West portion of the reservoir, far from the oil rim. Although not that much physical, overshooting did not bias oil in place computations but simply gave pressure support.

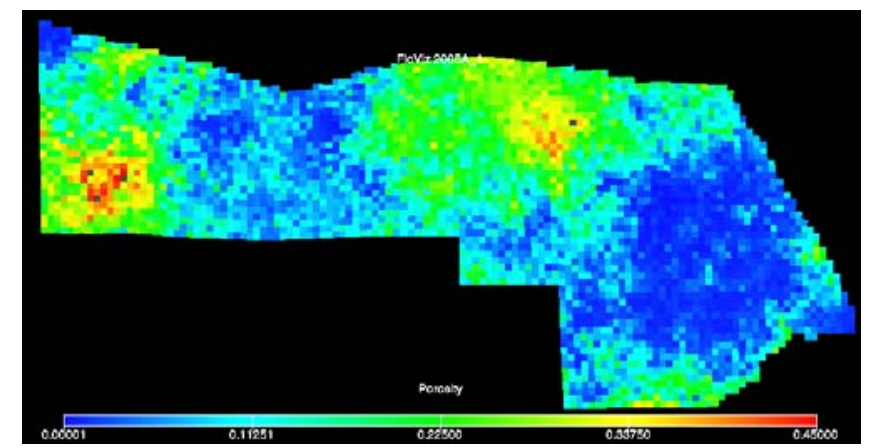

Figure 11: Average porosity for ensemble $A$ after EnKF integration in a representative layer.

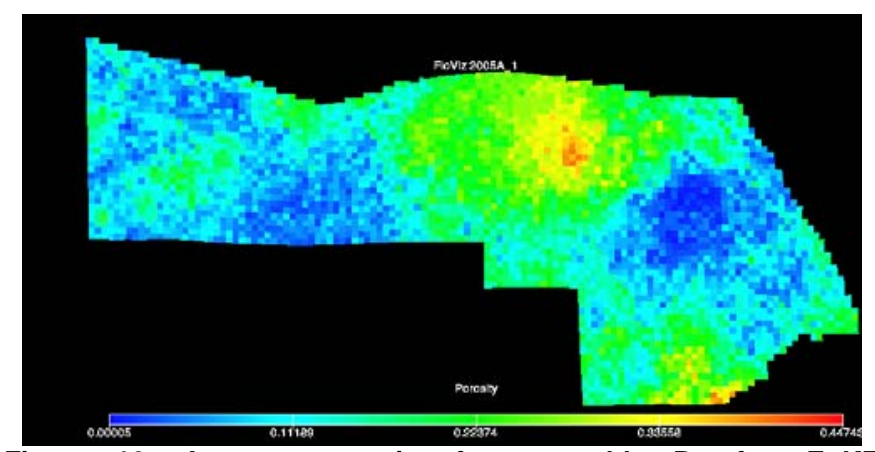

Figure 12: Average porosity for ensemble $B$ after EnKF integration in a representative layer.

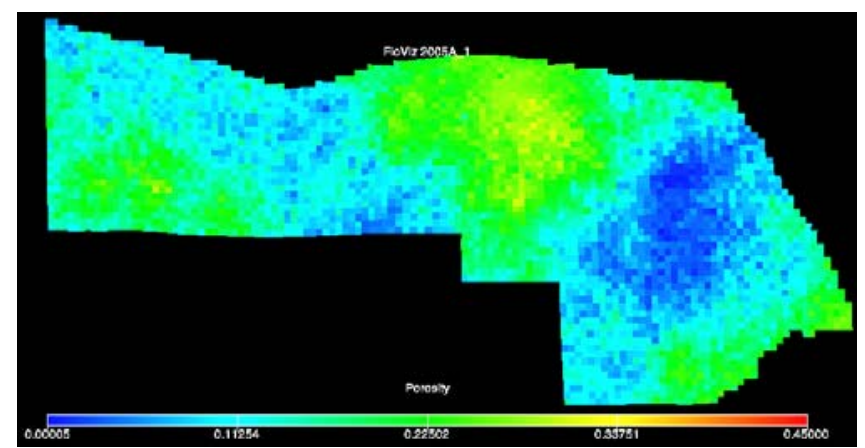

Figure 13: Average porosity for ensemble $C$ after EnKF integration in a representative layer.

The EnKF is a Monte Carlo methodology, aimed at sampling a posterior probability distribution. Hence, from a theoretical point of view it is somehow inappropriate to look for a single model, able to represent the integrated ensembles. Nonetheless, for many practical reasons, in history matching projects it is often required to give a reference model, to be used for deterministic purposes like defining reserves, quantifying the net present value of the field or provide guidelines for a wells infilling program. In this framework, the best candidates to represent the integrated ensembles are the models defined by the ensemble means, that is to say $\left\langle m_{s}\right\rangle_{A}^{u}$, $\left\langle m_{s}\right\rangle_{B}^{u}$, and $\left\langle m_{s}\right\rangle_{C}^{u}$.

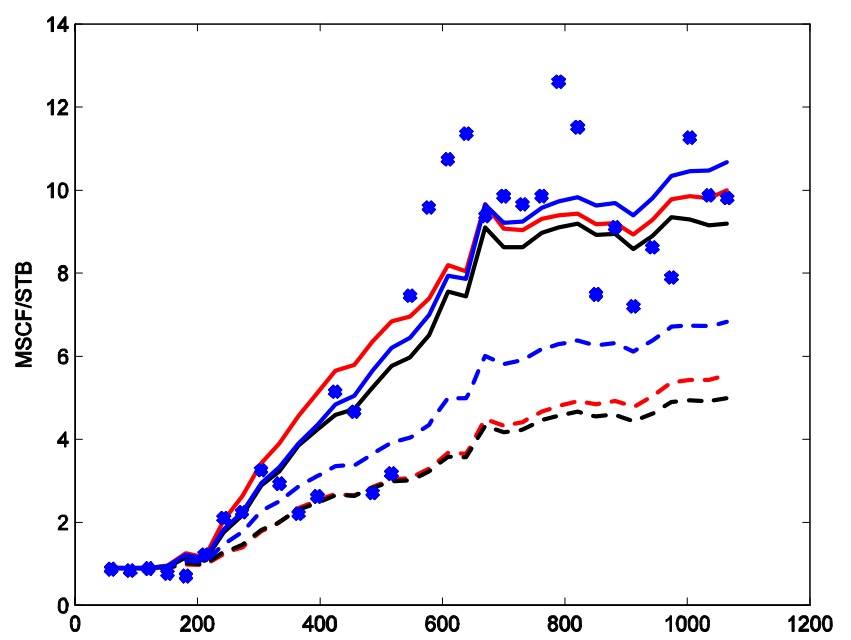

Figure 14: Well A1. Measured GOR (blue crosses) values and simulated GOR values (lines). Dashed lines correspond to average porosity fields before EnKF integration, continuous lines correspond to average porosity fields after EnKF integration. Black, red and blue correspond to ensemble A, B, and C, respectively.

Figure 14 shows GOR values computed on the basis of integrated porosity mean values $\left\langle m_{s}\right\rangle_{A}^{u},\left\langle m_{s}\right\rangle_{B}^{u}$, and $\left\langle m_{s}\right\rangle_{C}^{u}$, together with original porosity mean values $\left\langle m_{s}\right\rangle_{A}^{O}$, $\left\langle m_{s}\right\rangle_{B}^{o}$, and $\left\langle m_{s}\right\rangle_{C}^{o}$. This picture indicates that also the mean models can be considered individually history matched models, suitable for deterministic-type purposes.

\section{Ensemble Standard Deviation Updates}

In this section the key issue is to evaluate how EnKF operated on the ensemble uncertainty in the porosity field. 
Geostatistics, or any kind of stochastic approach to reservoir characterization, is implemented to quantify the uncertainty due to the limited amount of information available on the reservoir. The purpose of any stochastic history matching workflow is then to make the model less uncertain. In the case of Zagor reservoir, the uncertainty in the parameterization could be quantified in terms of the variation of the standard deviation for the ensembles.

Likewise ensemble mean fields, see equation (9), a field porosity standard deviation $\left\langle\sigma_{s}\right\rangle$ could be defined by computing cell-wise standard deviations over the ensemble members. The update operated by EnKF on the standard deviation fields can be evaluated by means of the maps shown from Figure 15 to Figure 17 for the same representative layer seen in Figures 11-13. The three figures are organised as follows: the standard deviation field for the original ensemble is shown in the top row, while the bottom row shows the standard deviation field for the integrated ensemble. The former plot reflects the prior uncertainty in the parameter estimation; the latter plot reflects the posterior uncertainty. It is worth noticing that prior uncertainty is low in the East part of the reservoir, where the wells intersect the reservoirs. Wells positions are defined in the maps by the white spots, corresponding to zero standard deviation values.

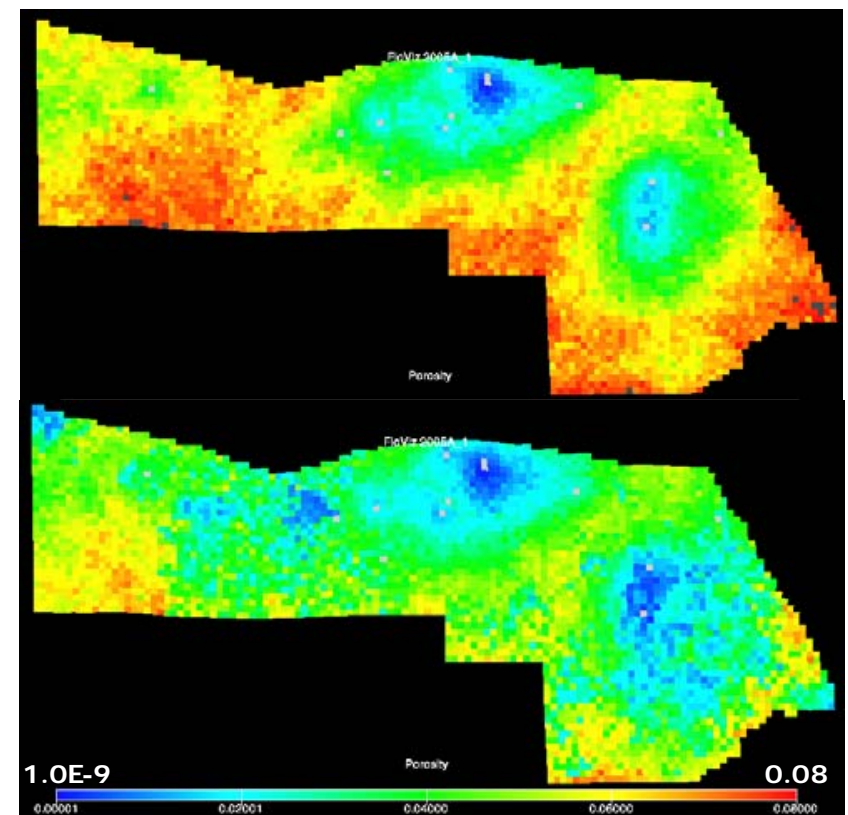

Figure 15: Ensemble A - Porosity standard deviation, in a representative layer, before (top row) and after EnKF (bottom row) assimilation. Red means values around 0.08 , blue values close to 0.

In the case of ensemble A, Figure 15, the uncertainty in the original ensemble - top row map - was spread toward the boundary of the reservoir, far from the wells. The EnKF update after 33 time-steps reduced systematically the uncertainty, with some very high residual uncertainty - red spots - in the South-East flank.

As regards ensemble B, see Figure 16, the effect of EnKF is similar to what already noticed for ensemble A. By comparing the top row map with the bottom row one it can be noticed a reduction in the uncertainty in the areas far from the wells (both production and appraisal ones). However, in some zones of the reservoir standard deviation values are higher for integrated ensemble B than for integrated ensemble A. This, together with the analysis of the porosity overshooting for the two ensemble may indicate that the integrated ensemble A represents the posterior distribution better than integrated ensemble B.

Moving the attention to the larger ensemble, that is to say ensemble $\mathrm{C}$, the result of the integration is qualitatively similar to what already noticed for the smaller ensembles: uncertainty reduced because of EnKF integration. Notably, the spatial distribution of the uncertainty after integration followed a trend going from ensemble A to C, from smaller to larger statistical samples. Areas close the West and South-East flanks became more uncertain going from smaller to larger statistical samples.

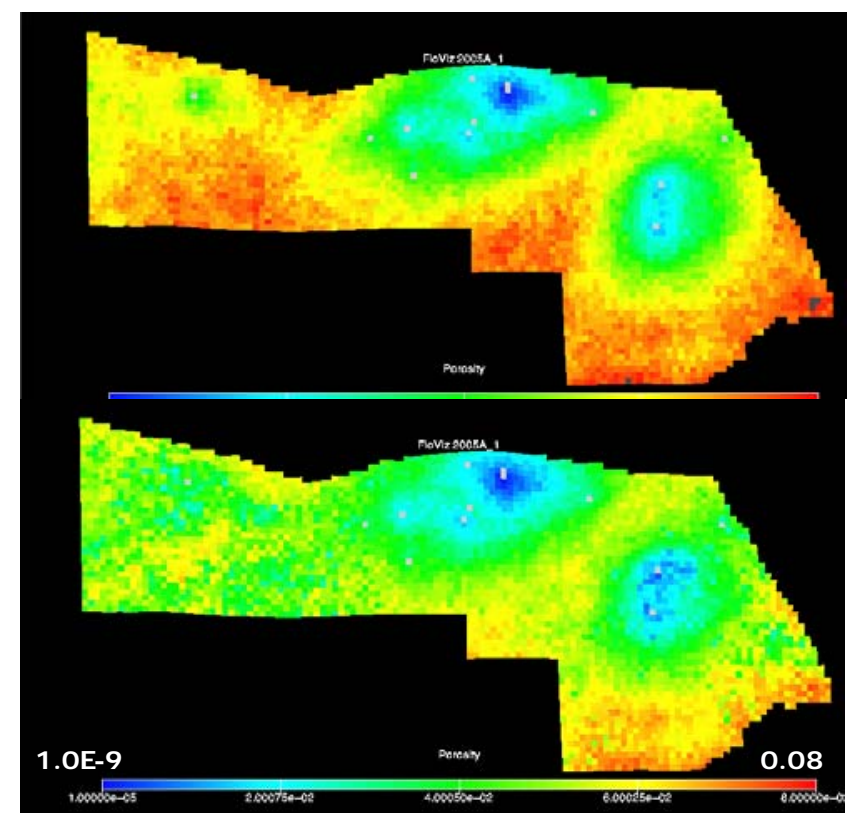

Figure 16: Ensemble B - Porosity standard deviation, in a representative layer, before (top row) and after EnKF (bottom row) assimilation.

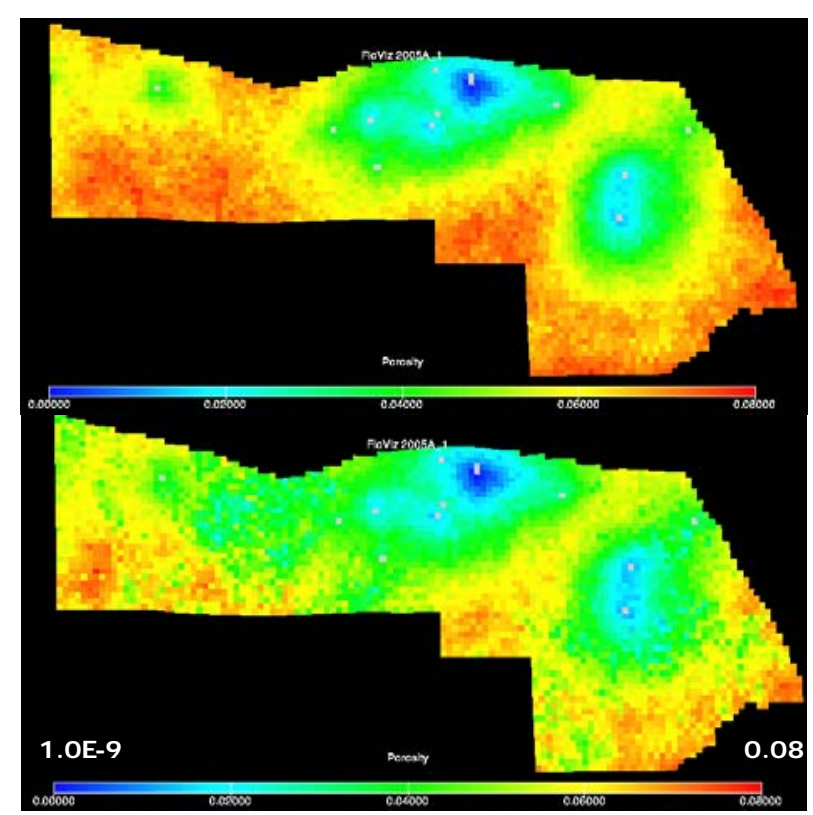

Figure 17: Ensemble C Porosity standard deviation, in a representative layer, before (top row) and after EnKF (bottom row) assimilation. 


\section{Production Forecasts Uncertainty}

To evaluate the uncertainty in the production forecasts, one of the ensembles, that is to say ensemble A, could be run in predictive mode to simulate 23 years of prediction. The prediction period was simulated by using a minimum tubing head target of 300 psia for both wells, with an abandonment constraint for economic reasons when WCT was higher than $60 \%$.

The uncertainty in the predictions can be evaluated by means of the data reported in Figure 18. The standard deviations in the predicted cumulative oil production were reduced by EnKF application, with a final $40 \%$ reduction at the end of the $23^{\text {rd }}$ year of forecast.

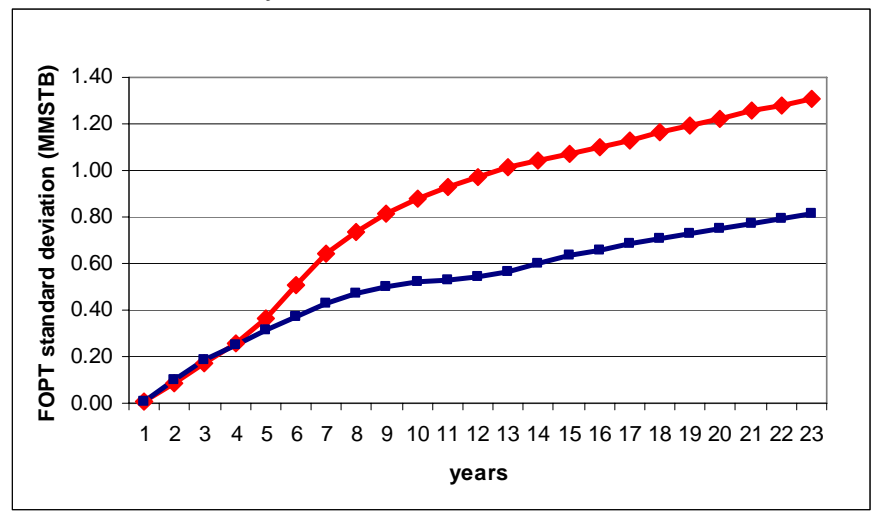

Figure 18: Cumulative field oil production standard deviation vs time for 23 years forecast. Red line corresponds to the original ensemble $A$ predictions, blue corresponds to the integrated ensemble A predictions.

In Figure 19 average incremental cumulative oil production was combined with the standard deviations shown in Figure 18. This figure indicates that the differences between original and integrated ensemble predictions are statistically significant.

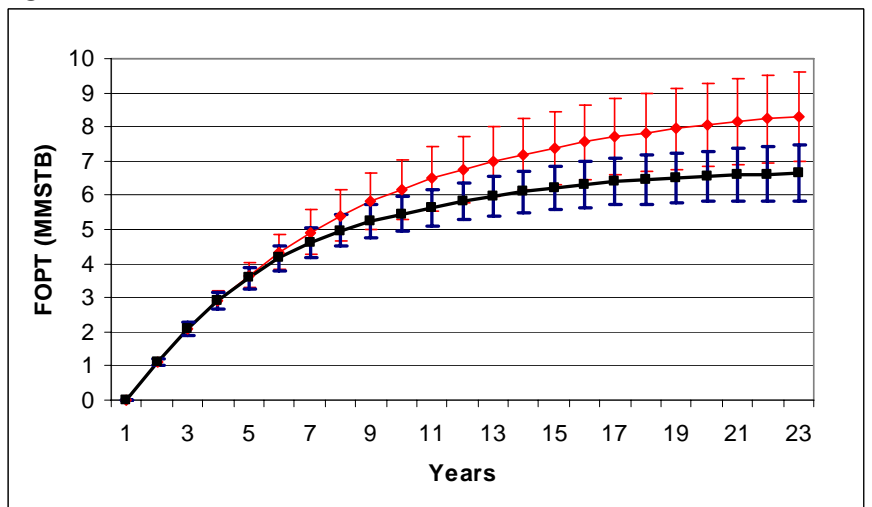

Figure 19: Average FOPT in a 23 years forecast for the ensemble A. Red line corresponds to the original ensemble A predictions, blue corresponds to the integrated ensemble $A$ predictions. Standard deviations were used to define the error bars.

\section{Conclusions}

In this work EnKF was used to calibrate porosity fields used to model an oil reservoir with almost three years of production. This confirmed the possibility to use EnKF for history matching real reservoir models.

In addition, an analysis of the dependence of EnKF effectiveness on the number of fields included in the statistical ensembles was performed by using three ensembles of 50, 100 and 135 members, respectively. Although this work represents the first attempt to investigate the connection between EnKF effectiveness and the size of the ensemble for a real problem, the limited number of ensembles considered did not give the chance to lead to some ultimate conclusion on key points like the number of fields required from a practical viewpoint. Nonetheless, some interim conclusions can be drawn.

First, this analysis indicates that, from a history matching point of view, the quality of the calibration could be improved by using ensembles with 100 or 135 members. That was quantitatively confirmed by means of the objective function values reported in Table 1, although the differences are not high.

Second, as regards statistics for porosity fields, differences could be noticed among the three ensembles, namely A, B, and $\mathrm{C}$, after EnKF integration. Mean porosity values indicated some local overshooting due to integration in the case of ensemble $\mathrm{A}$ and, with less evidence, also in the case of ensemble $\mathrm{B}$, while ensemble $\mathrm{C}$ was not affected by this problem. On the other side, investigations on porosity standard deviations fields after EnKF did not lead to clear conclusions. The expectation of having less uncertainty going from integrated ensembles A to $\mathrm{C}$ was not confirmed by visual inspections of the fields.

The possibility to use the integrated porosity ensembles to predict with less uncertainty the production of the field was proven in the case of ensemble A.

\section{Acknowledgments}

We acknowledge Eni Exploration and Production Division for the permission to publish this paper.

\section{References}

1. Evensen, G., "Sequential Data Assimilation with a nonlinear quasi-geostrophic model using Monte Carlo methods to forecast error statistics", J. Geophys. Res. Vol. 99, 10143-10162, 1994.

2. Naevdal, G., Mannseth, T., and Vefring, E., "Near-Well Reservoir Monitoring Through Ensemble Kalman Filter", SPE 75235, SPE/DOE Symposium on Improved Oil Recovery, Tulsa, Oklahoma, U.S.A., 13-17 April, 2002.

3. Naevdal, G., Johnsen, L. M., Aanonsen, S. I., and Vefring, E., "Reservoir Monitoring and continuous model updating using ensemle Kalman filer", paper SPE 84372, SPE Annual Technical Conference and Exhibition, Colorado, U.S.A., Denver, 5-8 October, 2003.

4. Gu, Y., and Oliver D. S., "The Ensemble Kalman Filter for Continuous Updating of Reservoir Simulation Models", Journal of Energy Resources Technology, Volume 128, Issue 1, pp. 79-87, March 2006.

5. Zafari, M., and Reynolds, A., "Assessing the Uncertainty in Reservoir Description and Performance Predictions with the Ensemble Kalman Filter", SPE 95750, SPE Annual Technical Conference and Exibition, Dallas, Texas, U.S.A., 9-12 October, 2005.

6. Wen X. H., and Chen W. H., "Real-Time Reservoir Model Updating Using Ensemble Kalman Filter", SPE 92991, SPE Reservoir Simulation Symposium, Houston, Texas U.S.A., 31 January-2 February, 2005.

7. Gu Y., and Oliver D. S., "History Matching of the PUNQS3 reservoir model using the ensemble Kalman filter", paper SPE 92867, SPE Annual Technical Conference and Exibition, Houston, 26-29 September, 2004.

8. Oliver, D. S., "On Conditional Simulation to Inaccurate Data", Math. Geology, 28(6), 811-817, 1996.

9. Liu, N., and Oliver, D. S., "Evaluation of Uncertainty assessment methods", SPE Journal, 8(2), 188-195, 2003. 
10. Bianco, A., "Application of ensemble Kalman filter (EnKF) methodology for history matching and risk analysis", Master's thesis, Imperial College, London, 2006.

11. He, N., Reynolds, A. C., and Oliver. D. S., "Threedimensional reservoir description from multiwell pressure data and prior information", Soc. Pet. Eng. J., pages 312327, 1997.

12. Skjervheim, J.-A., Evensen, G., Aanonsen, S. I., Ruud, B. O., and Johansen, T. A. "Incorporating 4D seismic data in reservoir simulation models using ensemble Kalman filter." Paper SPE 95789, presented at SPE Annual Technical Conference and Exhibition, Dallas, Texas, U.S.A., 2005.

13. Haugen, V., Natvik, L. J., Evensen., G., Berg, A., Flornes, K., and Naevdal, G., "History Matching Using an Ensemble Kalman Filter on a North Sea Field Case", SPE 102430, SPE Annual Technical Conference and Exhibition, 24-27 September, San Antonio, Texas, U.S.A., 2006.

14. Deutsch, C. V., and Journel, A. G., "GSLIB Geostatistical Software Library and User's Guide", Oxford University Press, 1998.

15. Houtekamer, P. L. and Mitchell, H. L., "Data assimilation using an ensemble Kalman filter technique." Monthly Weather Review, 126:796--811, 1998.

16. Thulin, K., and Naevdal, G., "Ensemble Kalman Filter for Field Estimation - Investigations on the Effect of the Ensemble Size", ECMOR X, Amsterdam, 2006.

17. Evensen, G., "Data Assimilation - The Ensemble Kalman Filter", Springer, 2006.

18. Lorentzen, R. J., Nævdal, G., Vallès, B., Berg, A. M., and Grimstad, A.-A., "Analysis of the ensemble Kalman filter for estimation of permeability and porosity in reservoir models", SPE 96375, SPE Annual Technical Conference and Exhibition, 9-12 October, Dallas, Texas, U.S.A., 2005. 